\title{
Retraction
}

\section{Characterization of giant spheroplasts generated from the aerobic anoxygenic photosynthetic marine bacterium Roseobacter litoralis}

\author{
Akane Nojiri, Shinjiro Ogita, Yasuhiro Isogai, and Hiromi Nishida \\ Biotechnology Research Center and Department of Biotechnology, Toyama Prefectural University, \\ Imizu, Toyama 939-0398, Japan \\ J. Gen. Appl. Microbiol., 61, 44-49 (2015) doi 10.2323/jgam.61.44
}

DNA extracted from the bacterial culture used in this paper was analyzed by the massively parallel DNA sequencing. The sequence similarity search showed that most of the obtained DNA sequences were similar to those of Enterobacter genomic DNA. PCR analyses using primers to detect Enterobacter DNA showed that the original Roseobacter culture was contaminated with this bacterium. The authors recognized that the cultures used in this paper were considered as a mixture of Enterobacter and Roseobacter litoralis, and the deduced results were not well rationalized. Thus, the JGAM editorial board agreed to retract the paper. 